\title{
Variability
}




\title{
HIGH-ENERGY CONTINUUM VARIABILITY
}

\section{IN ACTIVE GALACTIC NUCLEI}

\author{
RICK EDELSON \\ Department of Physics and Astronomy; University of Iowa; Iowa City, IA 52242; U.S.A.
}

September 30, 1993

\begin{abstract}
C G R O$ and IUE observations suggest that the strong, aperiodic variability seen in the Exosat long-look observations of AGN extends over a much wider energy band. Some BL Lac objects (but no Seyfert 1 galaxies) have shown X-ray variations which were so rapid that they violate the assumptions of isotropy inherent in the Eddington limit. In the ultraviolet, Seyfert $1 \mathrm{~s}$ as a class show an anti-correlation between the variability amplitude and luminosity, while BL Lacs show a positive correlation. Furthermore, Seyfert $1 \mathrm{~s}$ show strong flux-correlated spectral variability, while BL Lacs show little or none. All of this suggests that the high-energy continua of BL Lacs are beamed towards us, while the ultraviolet continua of Seyfert 1s are emitted isotropically.

The November 1991 multi-waveband monitoring of the BL Lac PKS 2155-304 showed strong correlated variability, with the soft X-rays leading the ultraviolet by a few hours, and no measurable lag between the ultraviolet and optical down to a limit of $\leqslant 1.5 \mathrm{hr}$. This indicates that the $\mathrm{X}$-rays from this BL Lac are not produced by Compton upscattering, and that the ultraviolet does not come directly from a thermal source such as an accretion disk. This also strongly constrains the relativistic jet model, suggesting that all of the radiation is produced in a flattened region like a shock front.

Low temporal resolution ultraviolet/optical monitoring of the Seyfert 1 NGC 5548 in 1989 yielded a strong correlation with no measurable lag to a limit of $\leqslant 4$ days, casting some doubt on the standard model of thermal emission from an accretion disk in Seyfert 1s. U.pcoming Xray/ultraviolet/optical monitoring of the Seyfert 1 NGC 4151 in December 1993 will have much faster sampling, to permit a strong test of both this model and the competing reprocessing model.
\end{abstract}

Key words: BL Lacertae Objects, $\gamma$-Rays, Quasars, Seyfert Galaxies, Ultraviolet, Variability, X-Rays

\section{Introduction}

Variability is emerging as a powerful tools for constraining the physics of Active Galactic Nuclei (AGN). Early single-band variability studies yielded constraints on the source sizes and energy densities that led to the original black hole/accretion disk picture (Rees 1984). Also, the observation of rapid radio variability, combined with the relatively low $\mathrm{X}$-ray fluxes provided the first strong evidence for beaming in flat spectrum radio sources, and led to predictions of superluminal motion that were later confirmed by VLBI (e.g., Pearson et al. 1981).

It has recently become possible to combine simultaneous observations taken at different wavebands (and with different telescopes), to construct single-epoch radio through X-ray (and, in some recent cases, through $\gamma$-ray) spectral energy distributions (SEDs) of AGN. These broadband "snapshots" have proven enormously valuable, suggesting what types of spectral components could contribute to different bands, thus providing a framework for formulating models of the physical

113

T. J.-L. Courvoisier and A. Blecha: Multi-Wavelength Continuum Emission of AGN, 113-122.

(C) 1994 IAU. Printed in the Netherlands. 
conditions and emission mechanisms operating in the centers of AGN (see, e.g., the contributions by Bregman and Maraschi in this volume).

However, such single-epoch SEDs cannot by themselves provide strong tests of these models. Model fits are underconstrained because they require a large number of free parameters, and experience has shown that a wide variety of models can produce acceptable fits. For example, both synchrotron self-Compton (SSC) models (e.g., Ghisellini, Maraschi \& Treves 1985) and accretion disk models (Wandel \& Urry 1991) have successfully been fitted to the broadband SED of the BL Lac object PKS 2155-304. Likewise, a variety of accretion disk models (Czerny \& Elvis 1987; Wandel \& Petrosian 1988; Madau 1988; Laor \& Netzer 1989; Sun \& Malkan 1989 , etc.) and free-free models (Barvainas 1993) provide adequate fits to Seyfert 1 SEDs.

Because the central regions of AGN are small and the spectral resolution and polarization capabilities of current satellites are limited, multi-waveband variability provides the best chance to resolve these ambiguities and constrain competing models. While this has long been recognized, only recently has there been the simultaneous availability of satellites operating at ultraviolet, X-ray and $\gamma$-ray wavelengths, the will to commit large amounts of telescope time, and the spirit of cooperation between large numbers of astronomers necessary to make it work.

Throughout this contribution, a distinction will be made between blazars (BL Lac objects and OVV quasars) and Seyfert 1 galaxies (and normal quasars). Also, while "high-energy" generally refers to the X-ray/ $\gamma$-ray regime, the broadband nature of AGN emission means that the ultraviolet and occasionally optical wavebands must be considered as well.

\section{Prevalence of Variability}

Strong, rapid, aperiodic high-energy variability is a common feature of AGN, as seen in Figure 1. The most rapid variations have been seen at $\mathrm{X}$-ray wavelengths, but this may be an artifact of the fact that Exosat, with its high Earth orbit (leading to long, uninterrupted light curves) and relatively high sensitivity, is particularly well-suited for studying AGN variability. By comparison, IUE, the workhorse of ultraviolet astronomy, can measure fluxes with high signal-to-noise ( $~(50 / 1)$, but has a minimum cycle time of $\sim 1 \mathrm{hr}$, and AGN have such low $\gamma$-ray count rates that $C G R O$ is generally only sensitive to $\gtrsim 10 \%$ variations on $\sim 1$ day time scales. Also, it is my impression that blazars show more rapid variations than Seyfert $1 \mathrm{~s}$, although no systematic comparison has ever been made.

The long-term ultraviolet light curves of blazars and Seyfert 1s look remarkably similar. This is probably due to the gross undersampling of the archival data, which are thus rendered insensitive to short time scale variability. Finally, with the discovery of a confusing source in the field of NGC 6814 (Madejski et al. 1993), there is no good evidence for periodicity in the light curve of any AGN. 

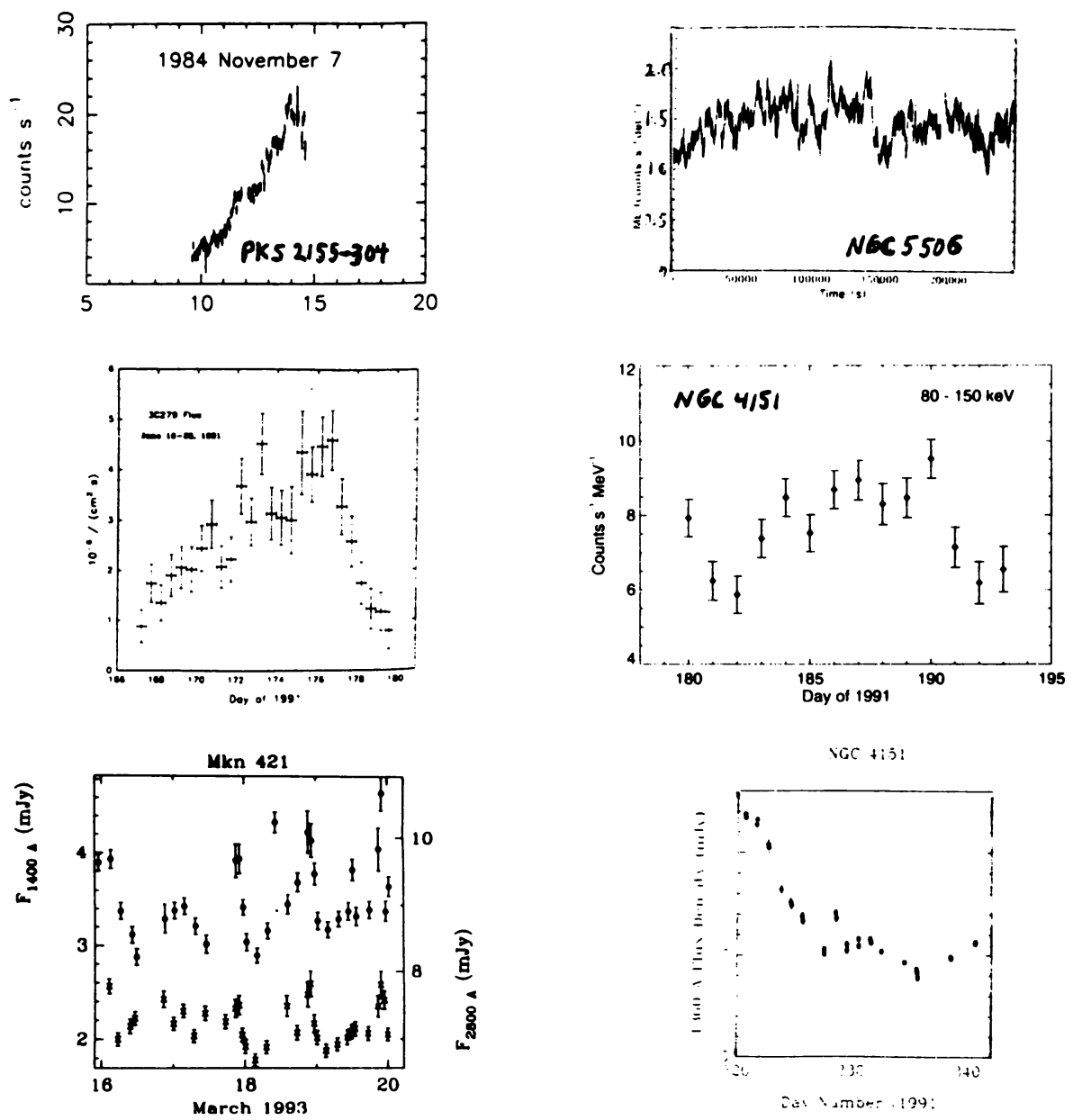

Fig. 1. Rapid variability of blazars (left) and Seyfert 1s (right) at X-ray (top), $\gamma$-ray (middle) and ultraviolet (bottom) energies. Data from Tagliaferri et al. (1991; Exosat ME observations of PKS 2155-304), Kniffen et al. (1993; CGRO EGRET, 3C 279), the ultraviolet archives (IUE SWP and LWP, Mkn 421), McHardy \& Czerny (1985; Exosat ME, NGC 5506), Maisack et al. (1993; CGRO OSSE, NGC 4151), the ultraviolet archives (IUE SWP, NGC 4151).

\section{Trends of Variability with Luminosity}

The earliest variability tests involved comparing the observed "doubling time" $\left(t_{1 / 2}=d t / d \ln F\right)$ with the minimum light-crossing time for a source emitting at the Eddington limit $\left(t_{l c}\right)$. For a spherical, optically thick source, the minimum light-crossing time is (Cavallo \& Rees 1978; Fabian 1979): 


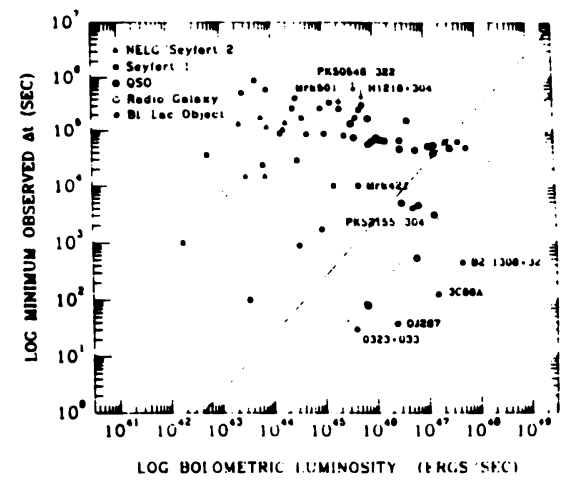

Fig. 2. Plot of most rapid observed X-ray variability doubling times as a function of bolometric luminosity (Bregman 1991). The solid line indicates the light crossing-time corresponding to a Schwarzschild radius of a system radiating a given Eddington luminosity. The dashed line corresponds to the maximum luminosity expected under the most conservative assumptions with asymmetric but unbeamed emission (Abramowicz \& Nobili 1982). All of the objects that lie below the line are blazars (BL Lacs or OVV quasars).

$$
t_{l c} \approx \frac{3 R_{s}}{c} \approx \frac{6 G \cdot M_{h}}{c^{3}} \approx \frac{L}{4 \times 10^{42} \mathrm{erg} \mathrm{s}^{-1}} .
$$

As seen in Figure 2. some blazars, but no Seyfert 1s, were found to vary more rapidly in the $X$-ravs than the minimum permitted light-crossing time. This is evidence that. as in the radio, the $\mathrm{X}$-rays from these blazars are not emitted isotropically, but instead are beamed towards us. The fact that so many Sevfert $1 \mathrm{~s}$ lie near the solid line supports the standard black hole model, since the only known way to radiate with this high efficiency is through gravitational liberation of the binding energy (Rees 1984). While this overall conclusion is probably still valid, the doubling time depends on the details of the observations (length of observation, sampling rate, and especially signal-to-noise) as well as intrinsic source properties, so it is risky to compare data taken with different telescopes or sampling patterns.

A possibly more robust approach involves examining variability amplitude of sources observed with a single telescope/detector system. Figure 3 shows a clear anti-correlation between long-term ultraviolet variability amplitude and mean source luminosity for the Seyfert 1s. This fits with the predictions of the model that the ultraviolet emission from Seyfert $1 \mathrm{~s}$ comes from an accretion disk (or some other relatively isotropically emitting source). The more luminous disks would be larger. with longer light-crossing times, and thus would vary more slowly.

There also appears to be a positive correlation between variability and both source luminosity and optical polarization for blazars. A simple explanation is again found in the context of beaming. A more strongly beamed source would have both the apparent luminosity and the degree of polarization boosted. and the apparent time scale would also increase. 

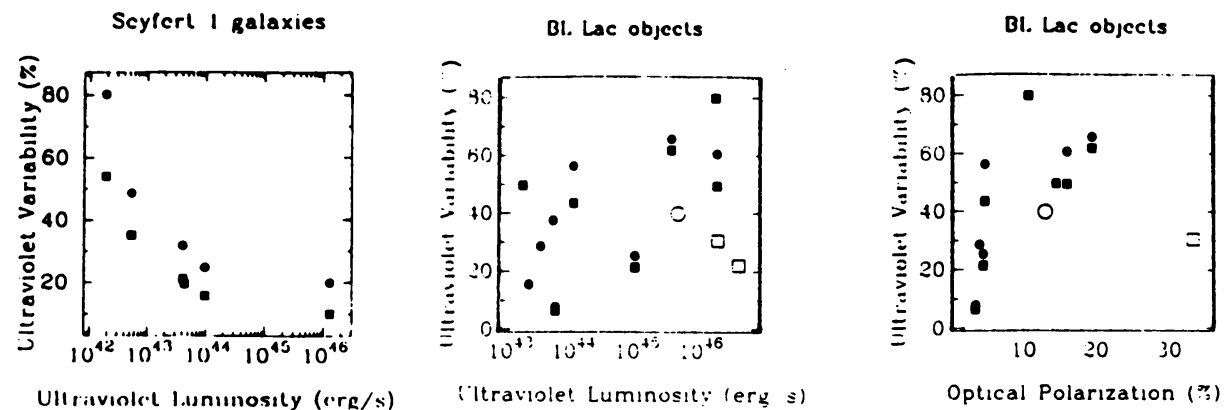

Fig. 3. Long-term ultraviolet variability plotted as a function of luminosity for Seyfert galaxies (left, Edelson, Krolik \& Pike 1990), and blazars (center, Edelson 1992). For blazars, variability is also plotted as a function of optical polarization (right). The circles refer to $1400 \AA$ data and the triangles to $2800 \AA$ data. The Seyfert 1 data (on the left) shows a clear anti-correlation, while the blazar data show an apparent positive correlation of degree of variability with both luminosity and optical polarization, especially if one excludes the three open symbols, which refer to poorly observed sources that have a mean time between observations of $\sigma_{\mathrm{t}}<2 \mathrm{yr}$, compared with $\left\langle\sigma_{t}\right\rangle=4 \mathrm{yr}$ for the others.

\section{Spectral Variability}

Spectral variability can bridge the gap between single-band and multi-band variability studies. If the flux is seen to vary while the spectral shape remains constant, this would suggest that the flux in that waveband is dominated by a single component, and that the line-of-sight absorption (if any) does not vary significantly. Likewise, the most natural explanation of significant spectral changes, especially when linked to flux changes, is the interplay of two emission components with different spectral shapes and variability properties. Other possible explanations include variable absorption or a single component with a variable shape.

Figure 4 gives ultraviolet flux-spectral index plots for Seyfert 1 s and blazars. The blazars show almost no significant spectral changes, supporting the hypothesis that a single component contributes to their ultraviolet emission. However, the Seyfert 1 s show strong spectral changes which are well correlated with flux level. This suggests that there are two components emitting significant ultraviolet flux: a harder, more strongly variable component (the putative accretion disk?) and a softer, less variable one. Other explanations are less tenable: If absorption causes the spectral and flux changes, large changes in reddening indicators such as line ratios and the $2175 \AA$ absorption features would be expected (none are observed), while if it was a single optically thick thermal source with a variable temperature (around $10^{5} \mathrm{~K}$, to reproduce the shape of the ultraviolet spectrum), even very large variations would have difficulty producing the observed spectral changes, because $L \propto T^{4}$ and $\alpha_{U V}$ is measured well below the spectral peak. 

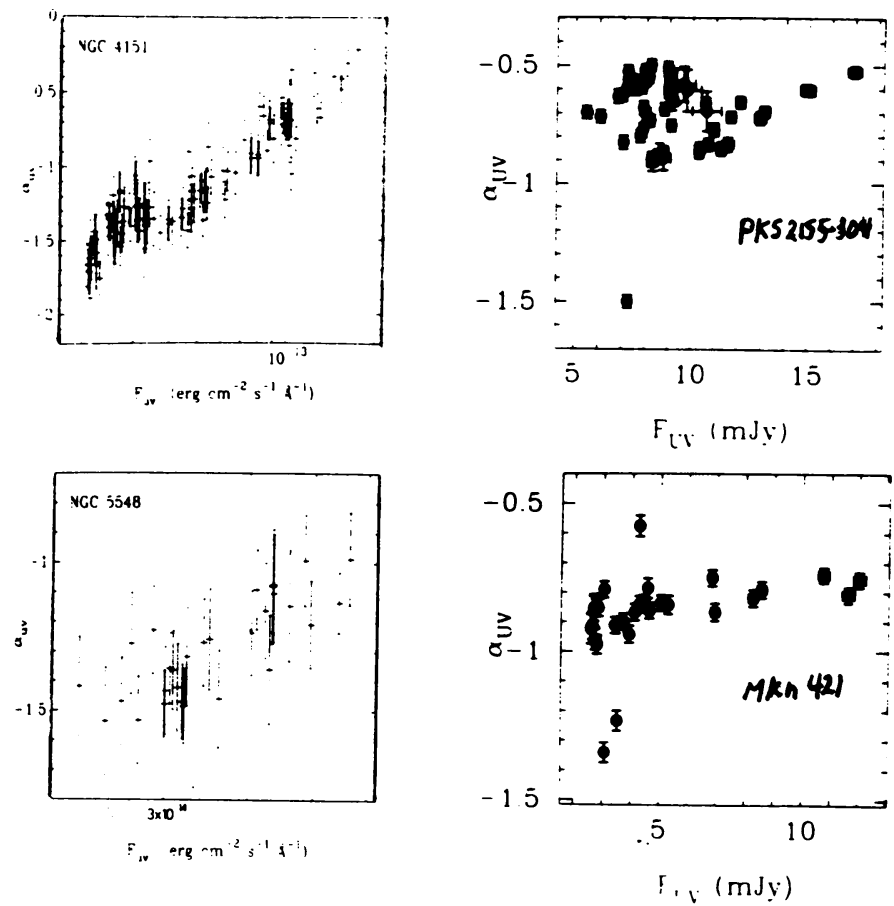

Fig. 4. Ultraviolet continuum spectral index, $\alpha_{U V}$ (defined as $F_{\nu} \propto \nu^{\alpha}$, measured between 1400 and $2800 \AA$ ) plotted against flux density for the two best observed Seyfert 1s (NGC 4151 and NGC 5548, on the left, from Edelson et al. 1990) and the two best observed blazars (PKS 2155-304 and Mkn 421, right, from Edelson 1992). For the Seyfert 1s, there is a strong correlation in the sense that the spectrum hardens as the source brightens, while for the blazars, there is little spectral variability and no evidence for a correlation between flux and spectral index.

The situation at higher energies is more uncertain, mostly because of the lack of sensitivity of low-resoluiton X-ray and $\gamma$-ray telescopes to relatively subtle spectral changes. The 2-10 keV spectra of Seyfert 1 s are generally thought to soften as the source gets brighter (e.g., Mushotzky, Pounds \& Done 1993), although NGC 7469 shows the opposite behavior (Barr 1986). This softening with increasing flux has also been observed in the soft $\gamma$-rays (Paciesas et al. 1993). In the soft X-rays (0.1$1 \mathrm{keV}$ ), there is some evidence for variations in the absorbing column, although multiple explanations are possible because of ambiguities introduced when fitting low-resolution X-ray spectra (e.g., intrinsic variability of the power-law slope: Yaqoob \& Warwick 1991; variability of the hard tail: Fiore et al. 1992; variable cold absorber: Bond, Matsuoka \& Yamanuchi 1993; variable warm absorber: Nandra et al. 1990). Because of its high resolution, the new X-ray satellite $A S C A$ will be very helpful in resolving such ambiguities. 

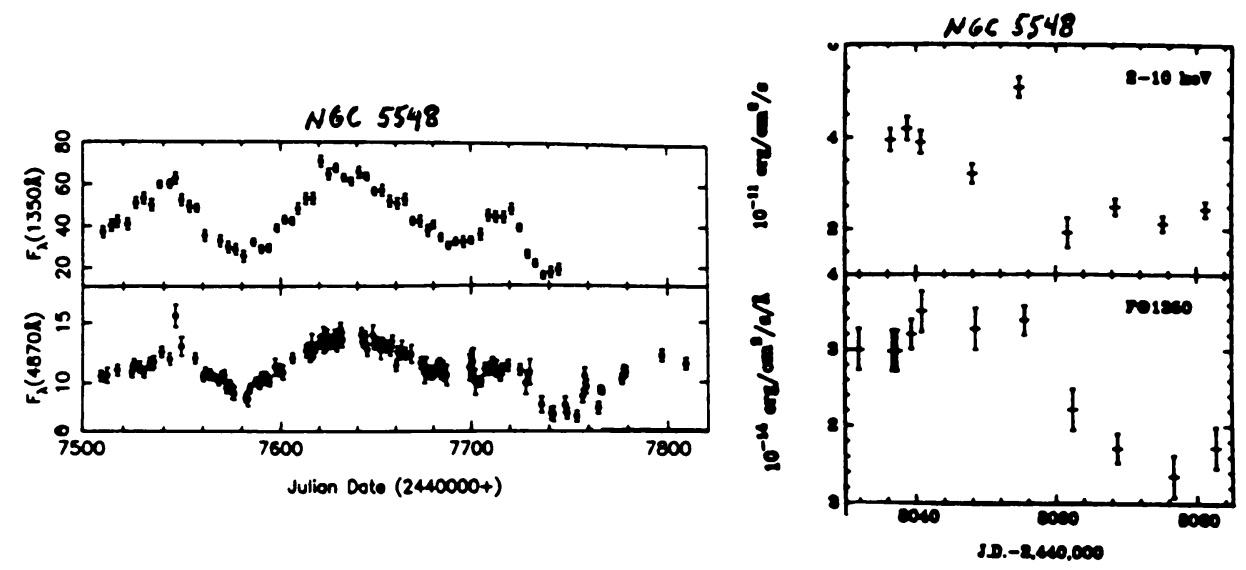

Fig. 5. Coordinated multi-waveband monitoring of the Seyfert 1 NGC 5548. On the left, ultraviolet/optical data show strongly correlated variations. The upper limit on the interband lag is $\leqslant 4$ days. On the right, ultraviolet/X-ray data taken at a different epoch suggest a correlation, although data taken previously do not fit this correlation.

\section{Multi-Wavelength Monitoring of NGC 5548}

The most constraining recent results resulted from intensive, coordinated monitoring of individual objects over a wide wavelength range. The first of these was the 1989 ultraviolet/optical campaign to monitor the Seyfert 1 NGC 5548 (Clavel et al. 1991; Peterson et al. 1991). Although the goal of this project was to map the structure of the broad emission "ine region, perhaps the most intriguing result came in the continuum, where it was found that there was no detectable lag between $1300 \AA$ ultraviolet and $5000 \AA$ optical variations, down to a limit of $\leqslant 4$ days (see Figure 5). In the standard accretion disk model, the $1300 \AA$ radiation is emitted at a mean radius of $\sim 0.1 \mathrm{lt}$-dy, while the $5000 \AA$ radiation is emitted at $\sim 0.5 \mathrm{lt}$-dy (Krolik et al. 1991), so the upper limit of $\leqslant 4$ days corresponds to a lower limit of the propagation speed of $\gtrsim 0.1 c$. This is much faster than either propagation at the sound speed or the orbital time scale. Similar results have been found for NGC 3783 (Reichert et al. 1993). The only mechanism left by which a signal can propagate at such a high speed is radiative reprocessing (Krolik et al. 1991), a scenario which had not been considered until recently. This potential problem with the accretion disk model has led to a renewed interest in alternative models, but a rigorous test awaits higher time sampling (see $\S 7$ ).

The most promising alternative to the accretion disk model is that the ultraviolet is reprocessed X-ray/ $\gamma$-ray radiation (e.g., Nandra et al. 1991). This model was supported by observation of an apparent correlation between ultraviolet and X-ray flux variations in NGC 5548 (Clavel et al. 1992; see Figure 5). Perola et al. 
(1986) found a similar possible correlation in NGC 4151. Such a correlation would be expected if the $\mathrm{X}$-rays drive the ultraviolet, although a clear test will again require much better sampling. Arguing against this model are: (1) the observed $\mathrm{X}$-ray/ $\gamma$-ray energy budget is not sufficient to produce the ultraviolet through infrared emission of higher luminosity quasars (although lower luminosity Seyfert 1s like NGC 5548 may not have this problem), and (2) no correlation was seen between X-rays and optical in other Seyfert 1s, most notably NGC 4051 (although a much shorter time scale was sampled; Done et al. 1990).

\section{Multi-Wavelength Monitoring of PKS 2155-304}

Coordinated X-ray/ultraviolet/optical observations have also strongly constrained emission from the blazar PKS 2155-304 (Edelson et al. 1993). As shown in Figure 6, it showed strong, highly correlated microvariability with the same amplitude in all bands. Careful correlation analysis showed that the X-rays led the ultraviolet by 2-3 hr, with no measurable lag between the ultraviolet and optical bands down to a limit of $\lesssim 1.5 \mathrm{hr}$. This important result rules out the hypothesis that the $\mathrm{X}$-rays are produced by SSC scattering of ultraviolet photons, since the $\mathrm{X}$-rays would have to come from a region as large or larger than the ultraviolet, and thus would be expected to track or lag the lower frequencies. Likewise, the claim that the ultraviolet is thermal emission from an accretion disk (Wandel \& Urry 1991) can also be rejected, both because a lag of $\gtrsim 50 \mathrm{hr}$ would be expected between $1400 \AA$ and $5000 \AA$ (by analogy to the argument made in the previous section), while none is seen, and because of the lack of spectral variability (see $\S 4$ ).

By process of elimination, this result supports the hypothesis that the entire optical through soft X-ray continuum arises from direct synchrotron emission in a jet. Broadband spectral fits based on the tapered jet model of Ghisellini, Maraschi \& Treves (1985) lead to very short lifetimes for electrons emitting in the X-rays $\left(t_{x} \lesssim 0.01 \mathrm{sec}\right)$, so the lack of the strong X-ray spectral variability (Brinkmann et al. 1993) means that they would have to be continuously re-accelerated in situ. This model also calls for a relatively large distance between the X-ray and optical emitting regions ( $\sim 10$ lt-day), which is difficult to reconcile with the observed $\mathrm{X}$-ray/optical lag of 2-3 hr, even after the effects of bulk relativistic motion are accounted for. Instead, this result favors a flattened geometry, such as that which would naturally arise in a shock front in which the X-ray and lower-energy emission are produced in essentially the same place (e.g., Marscher \& Gear 1985).

\section{Future Plans}

These initial large multi-waveband campaigns have been quite successful in eliminating models and constraining AGN physics, but there is clear potential for even greater progress in this rapidly evolving area. The aforementioned studies have taught us about the importance of evenly-spaced, high temporal sampling over 

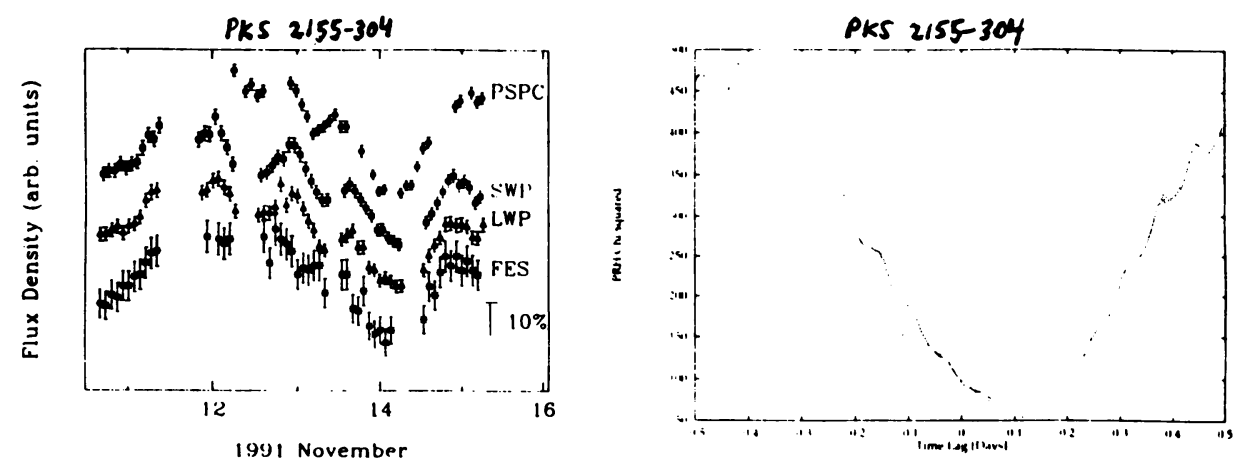

Fig. 6. Intensive monitoring of PKS 2155-304 at X-ray (top), $1400 \AA$ and $2800 \AA$ ultraviolet (middle) and $5000 \AA$ optical (bottom) energies (data from Brinkmann et al. 1993, Urry et al. 1993). The data show clear, correlated microvariability, with essentially no time lag between the ultraviolet and optical bands. The X-ray variations are also strongly correlated, but are $\sim 2-3 \mathrm{hr}$ ahead of the ultraviolet. On the right, correlation analysis using the Press, Rybicki \& Hewitt (1992) $\chi^{2}$ technique shows that this lag is significantly different from zero at the $5 \sigma$ level.

long time spans, good multi-waveband coordination, and careful source selection. For instance, even and rapid sampling is important because the erratic and aperiodic character of the variations will lead to aliasing if unevenly sampled, and the rapid nature of the variations means that the most valuable information will be lost if the sampling rate (or sensitivity) is too low.

The next round of campaigns has been constructed with these lessons in mind. The AGN Watch consortium (the group responsible for the NGC 5548 and NGC 3783 campaigns) will observe the rapidly variable Seyfert 1 NGC 4151 continuously on 1-10 December 1993, with IUE, Rosat, CGRO, ASCA and ground-based telescopes. Because of the fast sampling, multi-waveband coordination, and expected rapid source variability, this campaign should be able to measure ultraviolet lags so short that they would correspond to apparent propagation speeds that would exceed the speed of light, allowing an unambiguous test of the accretion disk model, and correlated ultraviolet/X-ray variations, to test the reprocessing model.

The blazar $0716+714$ will also be subjected to continuous, simultaneous IUE, Rosat, CGRO, ASCA and ground-based optical, infrared, submillimeter and radio monitoring over a 5.6 day period in March/April 1994. The goal of this campaign is to extend the PKS 2155-304 result to higher and lower frequencies, providing even more constraints on the geometrical relationships between wavebands. We also want to see if this radio-selected blazar behaves like the X-ray selected blazar PKS 2155-304. 
Beyond that, we hope to undertake one or two more multi-waveband AGN monitoring campaigns before IUE is turned off in September 1994. Terminating this functioning satellite will leave a gaping hole in the ultraviolet (the efficiency of $H S T$ is too low and its proposal pressure is too high to be of much use for such studies) and will deal a severe blow to the multi-waveband monitoring effort. Thus, this next year will see a flurry of activity in this area which will not be matched for the next few years. Let's hope we make good use of it!

\section{References}

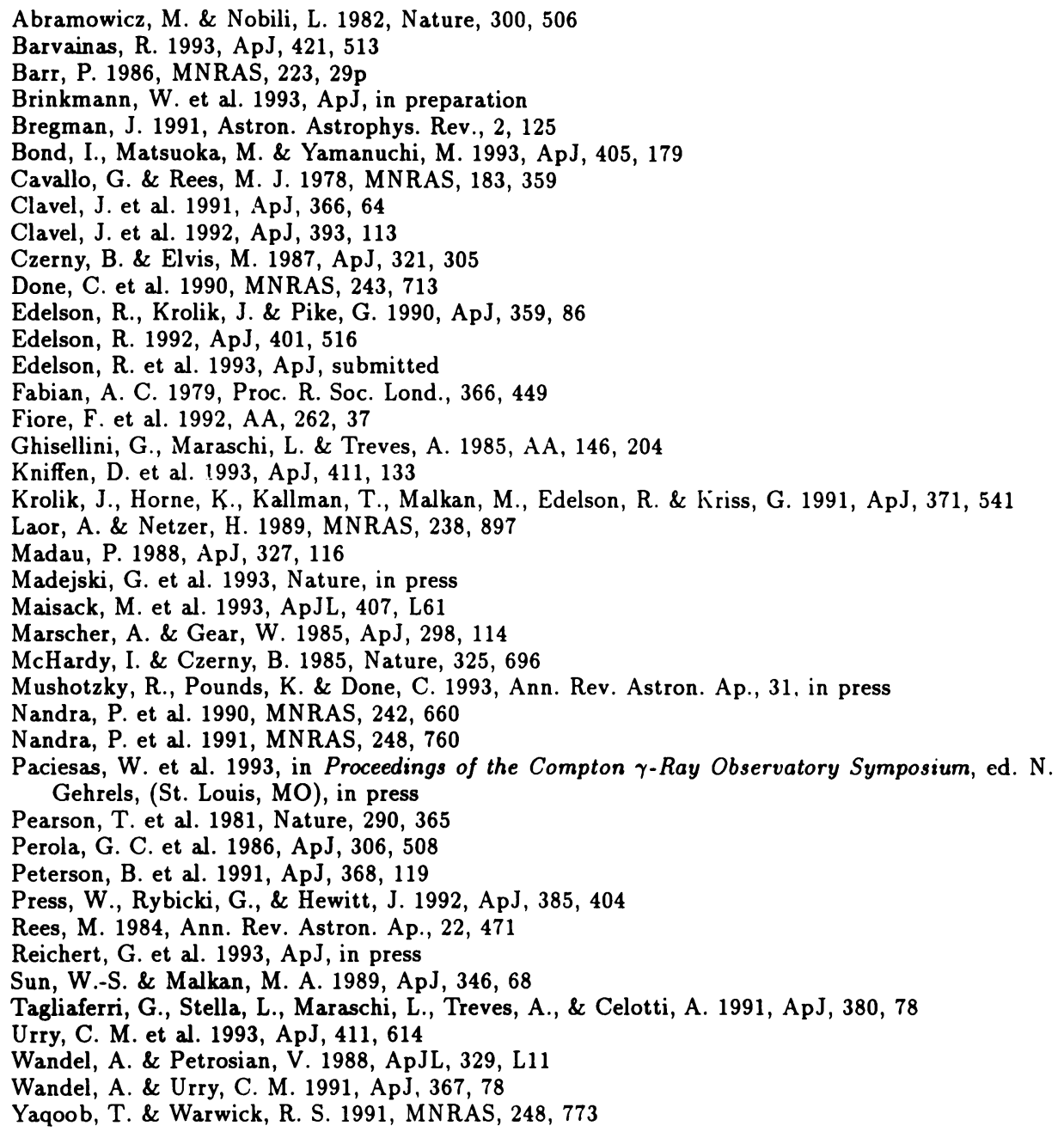

